\title{
Prototype Imaging Spectrograph for Coronagraphic Exoplanet Studies (PISCES) for WFIRST/AFTA
}

Qian Gong, Michael McElwain, Bradford Greeley, Bryan Grammer, Catherin Marx, Nargess Memarsadeghi, George Hilton, Marshall Perrin, Llop Sayson, Jorge Domingo, Karl Stapelfeldt

NASA Goddard Space Flight Center

Richard Demer, Hong Tang, Brian Kern, and Janan Ferdosi Jet Propulsion Laboratory 


\section{Overview}

- PISCES in JPL High Contrast Imaging Testbeds

- Comparisons to existing integral field spectrographs

- Selection of a lenslet-based IFS

- Lenslet demonstration and function of mask

- Relay optics and IFS optical design

- Trade off between reflective and refractive optics

- PISCES alignment plan 


\section{WFIRST Coronagraph and PISCES}

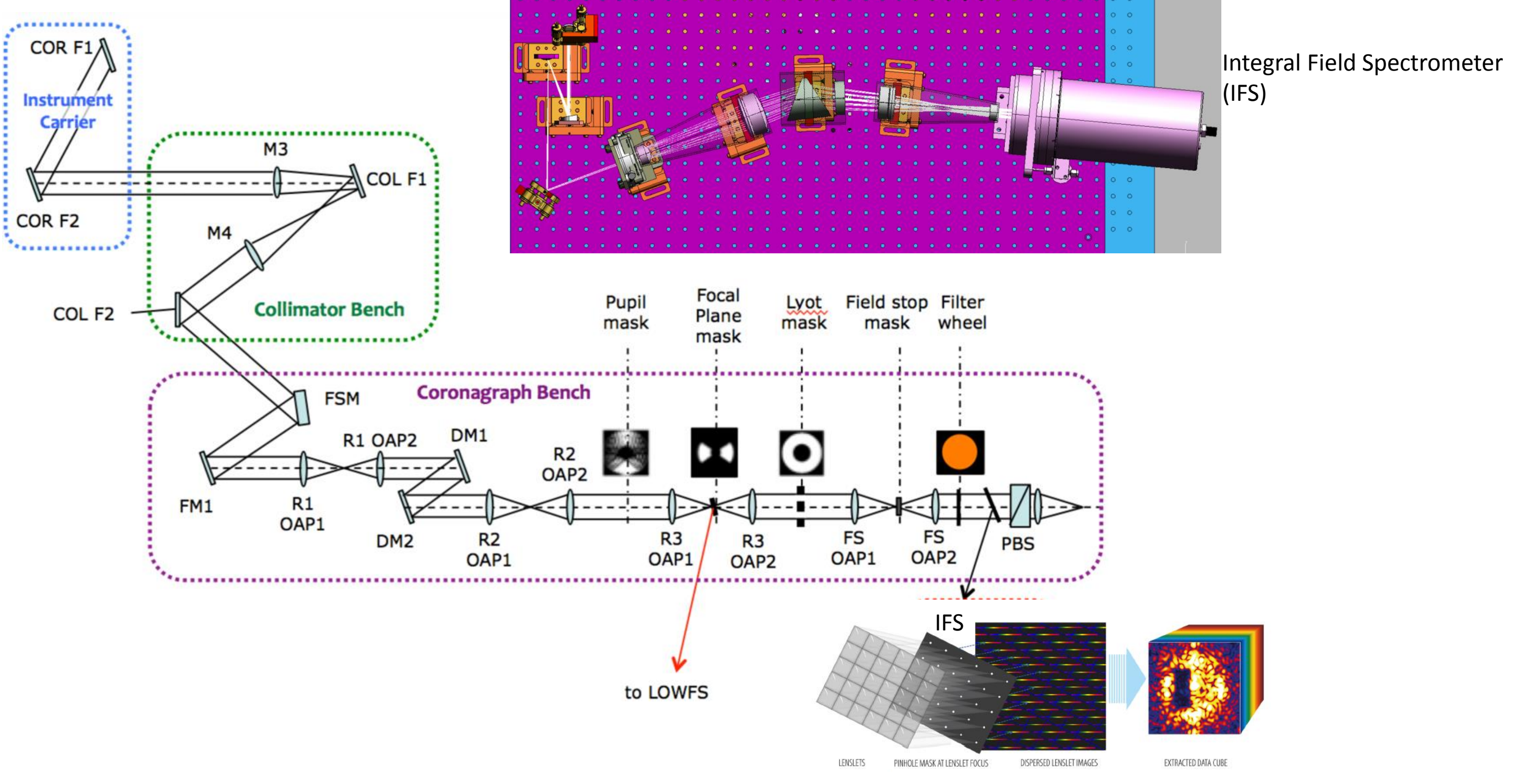




\section{IFS Designs Options}

IFS is a spectrometer that provides spectra at each spatial location in a 2D image. No matter which IFS, the goal is the same: to arrange space on the detector such that the spectra do not overlap.

Lenslets
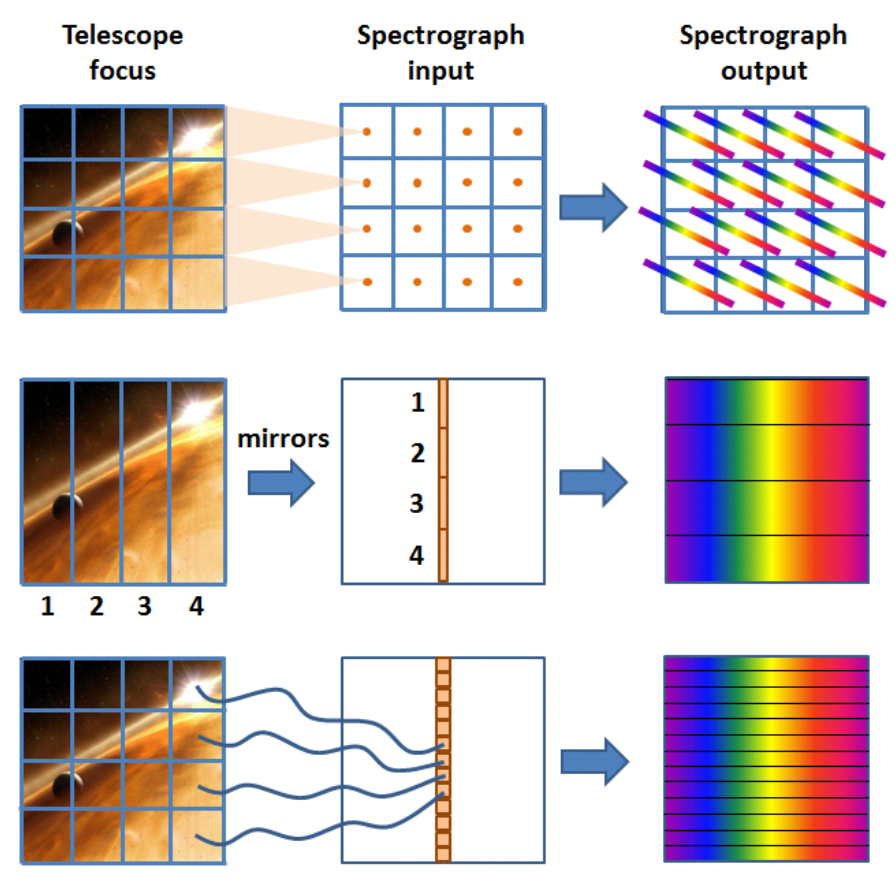
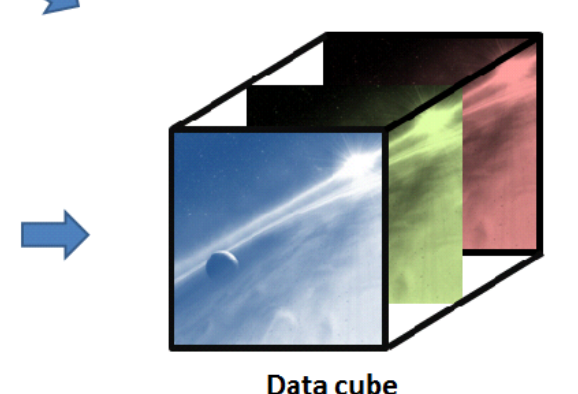

Data cube

\begin{tabular}{|l|l|}
\hline Pro & Con \\
\hline $\begin{array}{l}\text { Simplicity } \\
\text { High } \\
\text { throughput }\end{array}$ & Lower detector array usage efficiency \\
\hline $\begin{array}{l}\text { Efficient } \\
\text { detector array } \\
\text { usage }\end{array}$ & $\begin{array}{l}\text { Scattering loss } \\
\text { Additional optics is needed to arrange } \\
\text { slicers } \\
\text { Difficulty to fabricate high number of } \\
\text { slices }\end{array}$ \\
\hline $\begin{array}{l}\text { Efficient } \\
\text { detector array } \\
\text { usage }\end{array}$ & $\begin{array}{l}\text { Fiber coupling loss } \\
\text { Difficult to align and assemble fibers } \\
\text { Fiber material limits wavelength band }\end{array}$ \\
\hline
\end{tabular}




\section{Relay \& IFS Specification (As-designed)}

\begin{tabular}{|l|c|c|c|}
\hline \multicolumn{4}{|c|}{ Relay Specifications } \\
\hline $\begin{array}{l}\text { Central } \\
\text { wavelength (nm) }\end{array}$ & 660 & 770 & 890 \\
\hline$\lambda \min (\mathrm{nm})$ & 600 & 700 & 810 \\
\hline$\lambda \max (\mathrm{nm})$ & 720 & 840 & 970 \\
\hline $\begin{array}{l}\text { \# of dispersed } \\
\text { pixels }\end{array}$ & 26 & 26 & 26 \\
\hline f/\# & 870 & 870 & 870 \\
\hline Lenslet pitch & 174 & 174 & 174 \\
\hline Sampling at $\lambda_{\mathrm{c}}$ & 3.3 & 3.85 & 4.6 \\
\hline FOV (\# of $\lambda_{\mathrm{c}}$ /D) & 22.7 & 19.5 & 16.9 \\
\hline
\end{tabular}

\begin{tabular}{|l|c|}
\hline \multicolumn{2}{|c|}{ IFS Specifications } \\
\hline Wavelength range $(\mathrm{nm})$ & 660 \\
\hline Magnification & $1: 1$ \\
\hline $\mathrm{f} / \#$ & 8 \\
\hline Spectral resolution & $\mathrm{R}=70 \pm 5$ \\
\hline Spatial resolution & RMS spot diameter $<13 \mu \mathrm{m}$ \\
\hline Object size $(\mathrm{mm})$ & $13 \times 13$ \\
\hline Detector & $1024 \times 1024$ with $13 \mu \mathrm{m}$ pitch \\
\hline
\end{tabular}




\section{Selection of Lenslet-based IFS for PIECES}

- As mentioned in previous slides, the main advantage of the lenslet IFS is its simplicity and high throughput, the main disadvantage is the low efficiency of using detector pixels.

- For exo-planet coronagraph application, the Field Of Veiw (FOV) is small measured by $\lambda / D$, the dispersion (spectral resolution $R$ ) is low, so the low pixel efficiency is not a main concern.

- The operation wavelength range is from $600-1000 \mathrm{~nm}$. In this wavelength range, lenslet array has a very good transmittance. Besides, the material dispersion in that wavelength range is low enough to have the point spread function (PSF) diffraction limited in the entire wavelength range.

- The combination of lenslet array, pinhole mask, and none tele-centric relay optics design will further increase the coronagraph contrast. 


\section{Lenslet Array Design \& Demonstration}

- The lenslet array we have is basically the same as any other lenslet

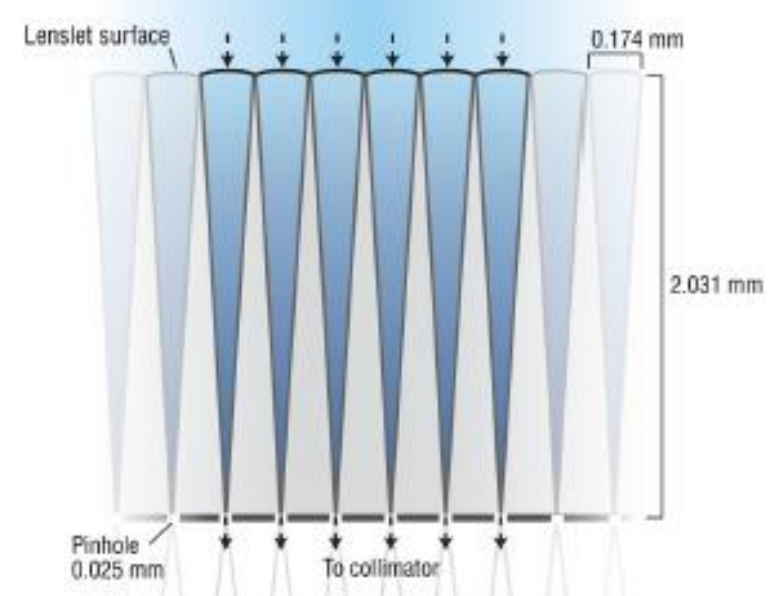
arrays except the followings:

1. A pinhole array mask is placed at the focal plane of the lenslet. The focal plane is at the back surface of the lenslet array, which guarantees the stability between lenslet and corresponding pinhole.

2. The function of the pinhole mask is to prevent the diffraction from lenslet edge to contaminate the PISCES spectra. However, it is also aimed to reduce the speckles of the coronagraph.

Lenslet Array Specification

Lenslet size

$174 \mu \mathrm{m} \times 174 \mu \mathrm{m}$

\# of lenslets

$76 \times 76$

$\mathrm{F} / \#$ in air 8 from side to side

Sampling $0.3 \lambda / \mathrm{D}$ at $600 \mathrm{~nm}$

Spectral channel 26

FOV

$25 \times 25 \lambda / \mathrm{D}$ at $600 \mathrm{~nm}$ $15 \times 15 \lambda / \mathrm{D}$ at $970 \mathrm{~nm}$

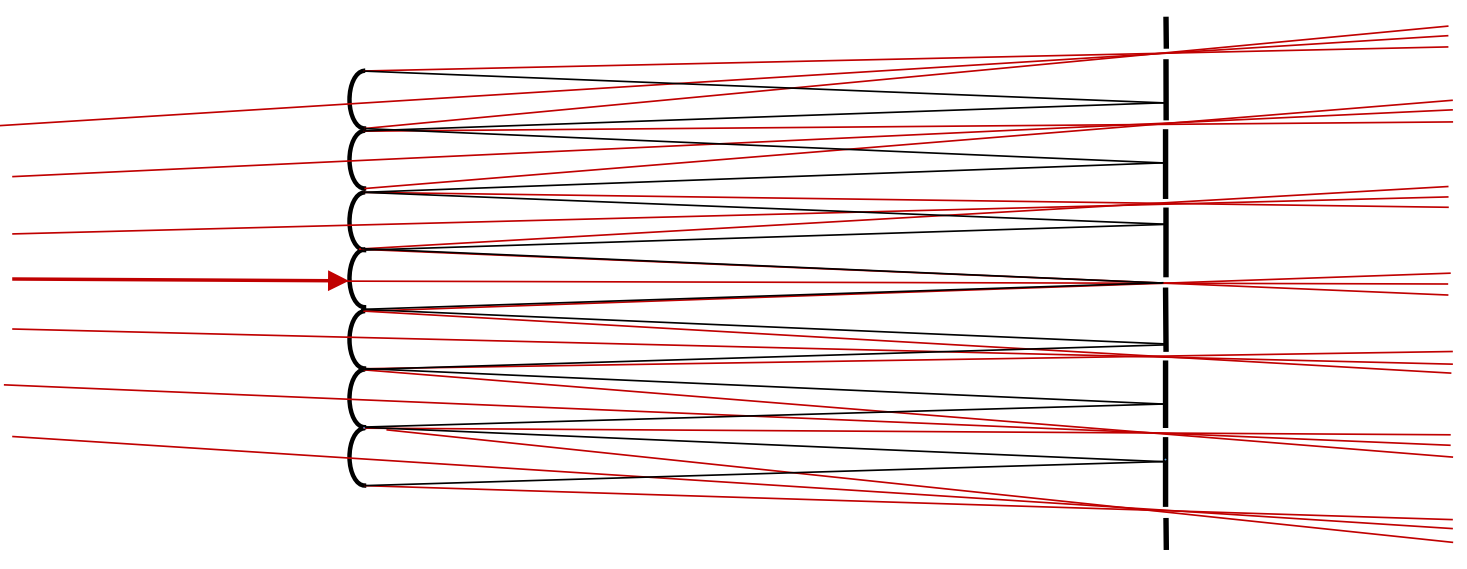

In this design, the relay is very non-telecentric, that is, the chief ray angle increases with the increase of the FOV. The pinhole positions are following the chief rays. As a result, the light (red rays) from the planets gets through, but the speckles (black rays) with relative flat wavefront are blocked by the mask. 


\section{Optical Design}

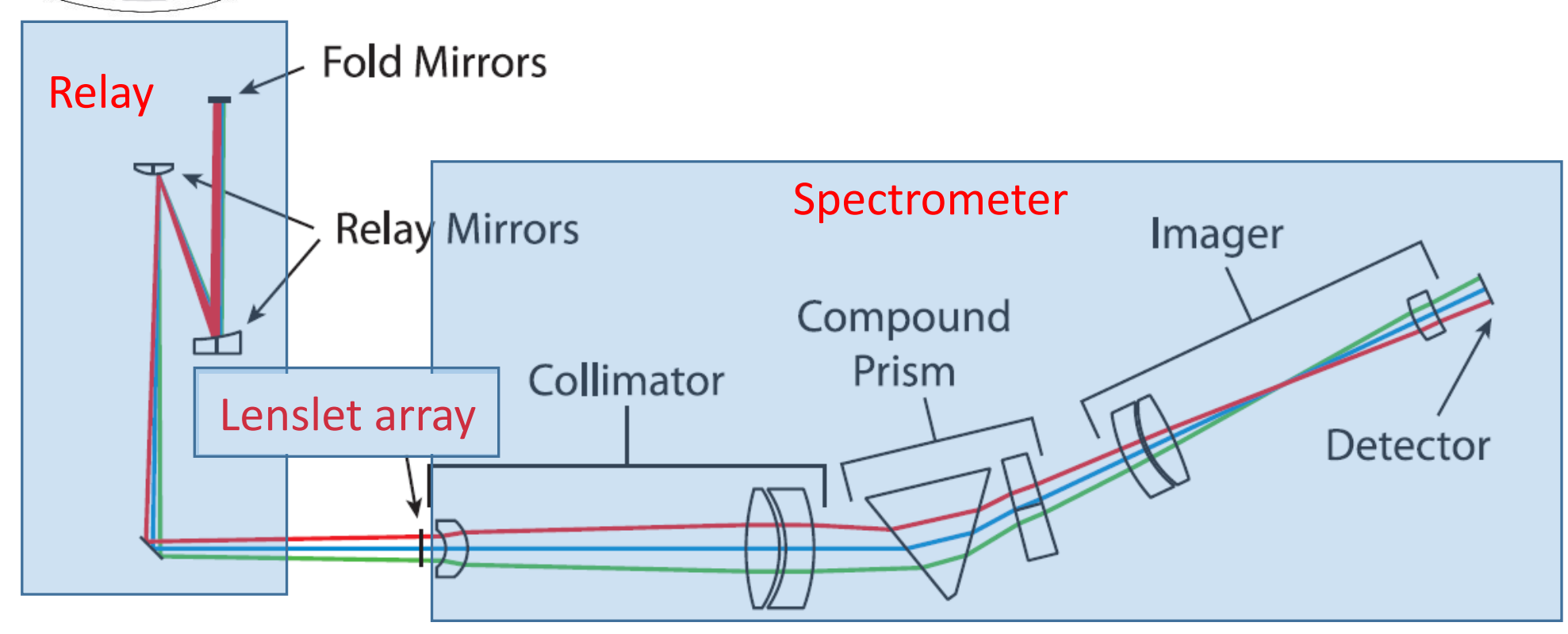

Optical design includes three parts:

1. Relay Optics - to adjust the plate scale to match required FOV into the designed lenslet array. The lenslet array dimension is determined by $n \lambda / D$ and sampling per $\lambda / D$, and the lenslet size is determined by spectral resolution, wavelength range, and the detector pixel size.
2. Lenslet array - to squeeze all light on one lenslet to a tiny spot in order to provide enough space to lay spectra.

3. Spectrometer - IFS spectrometer is similar to regular spectrometers except its multiple pupils. The number of the pupil is equal to the number of lenslets.

4. The spectrometer is consist of a collimator and a imager with a compound prism between them. 


\section{Dispersion Element Selection \& Design}

- Two most popular dispersive elements in spectrometers are gratings and prisms.

- However, the lenslet IFS prohibits using gratings. The reason is that the unwanted grating orders can't be eliminated from the detector array. They severely contaminate spectra of exo-planet. Therefore, a compound prism is selected as the dispersive element of the IFS spectrometer.

- The design options include single prism and compound prism, zero deviation and non-zero deviation. After a trade-off, the non-zero deviation compound prism is selected for obtaining most uniform spectral resolution across the entire wavelength range.

PISCES Spectral Resolution vs Wavelength

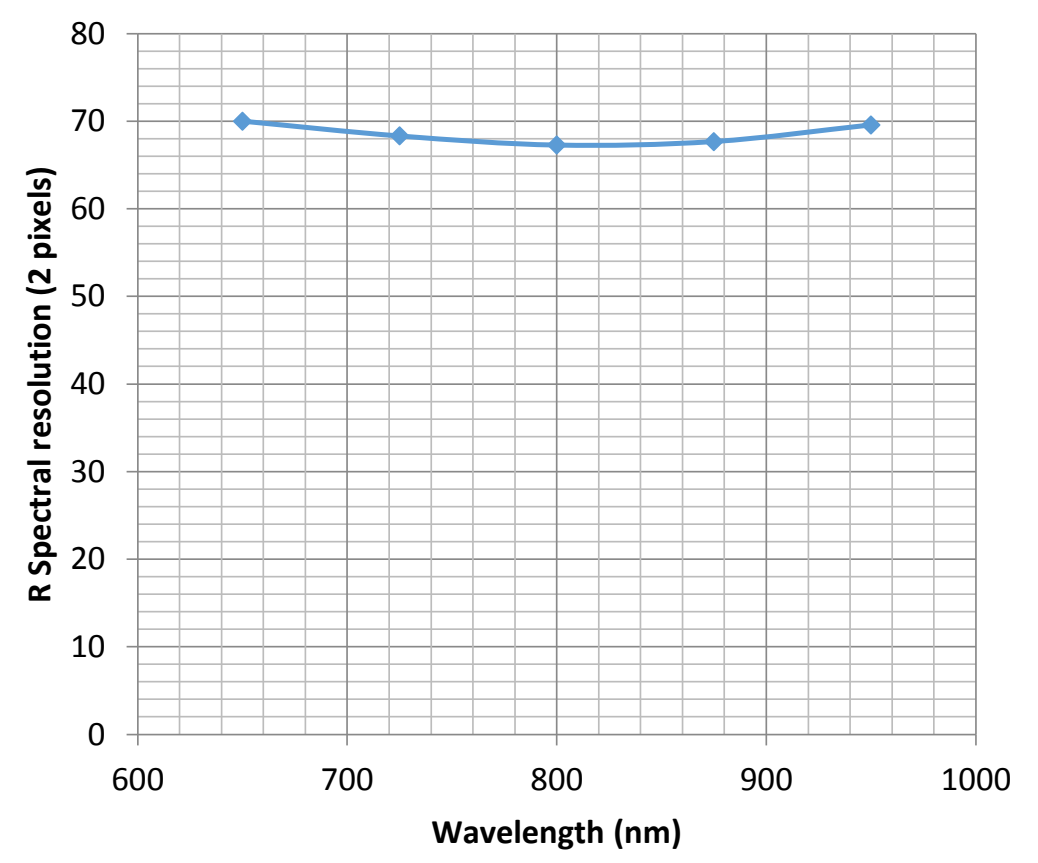

Spectral Resolution vs Wavelength For Different Options

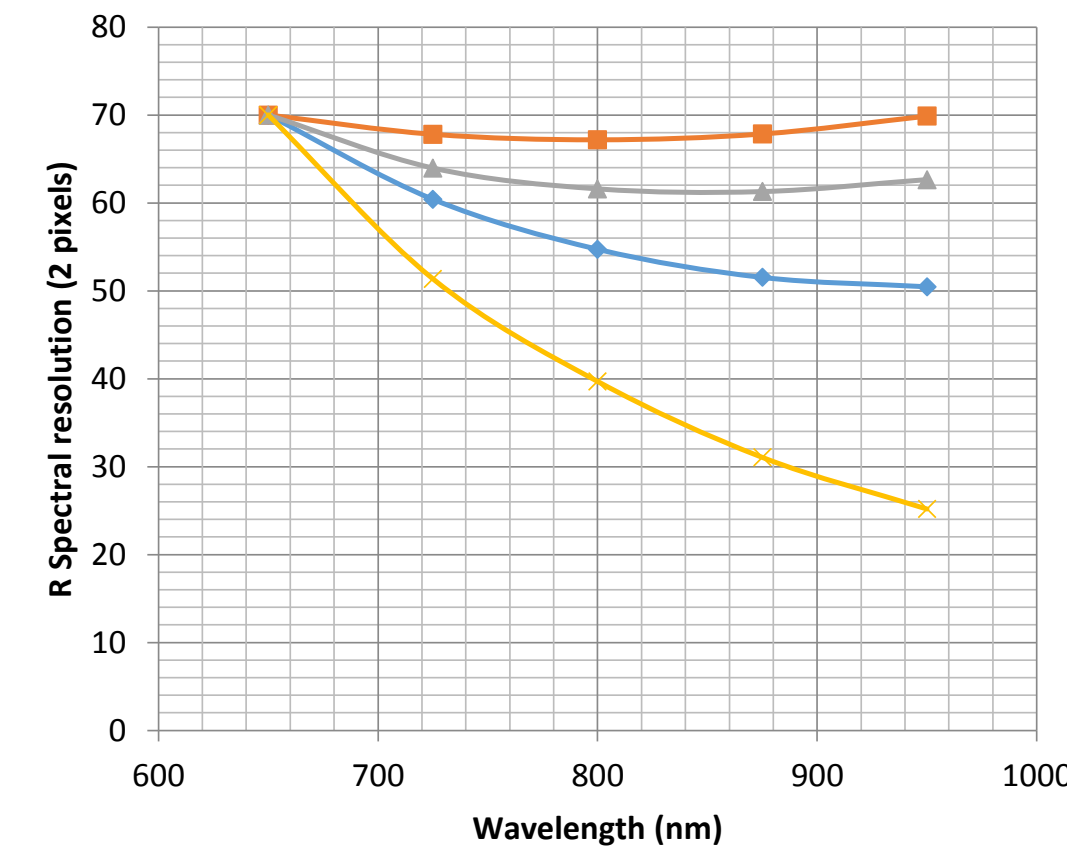

$\longrightarrow$ Non zero deviation Single SiO2

- Non zero deviation SiO2/ZnSe

$\leftarrow$ Non zero deviation SiO2/SF57

ZZero deviation YGH51/NPH1/YGH51 


\section{IFS Performance: Spatial and Spectral}

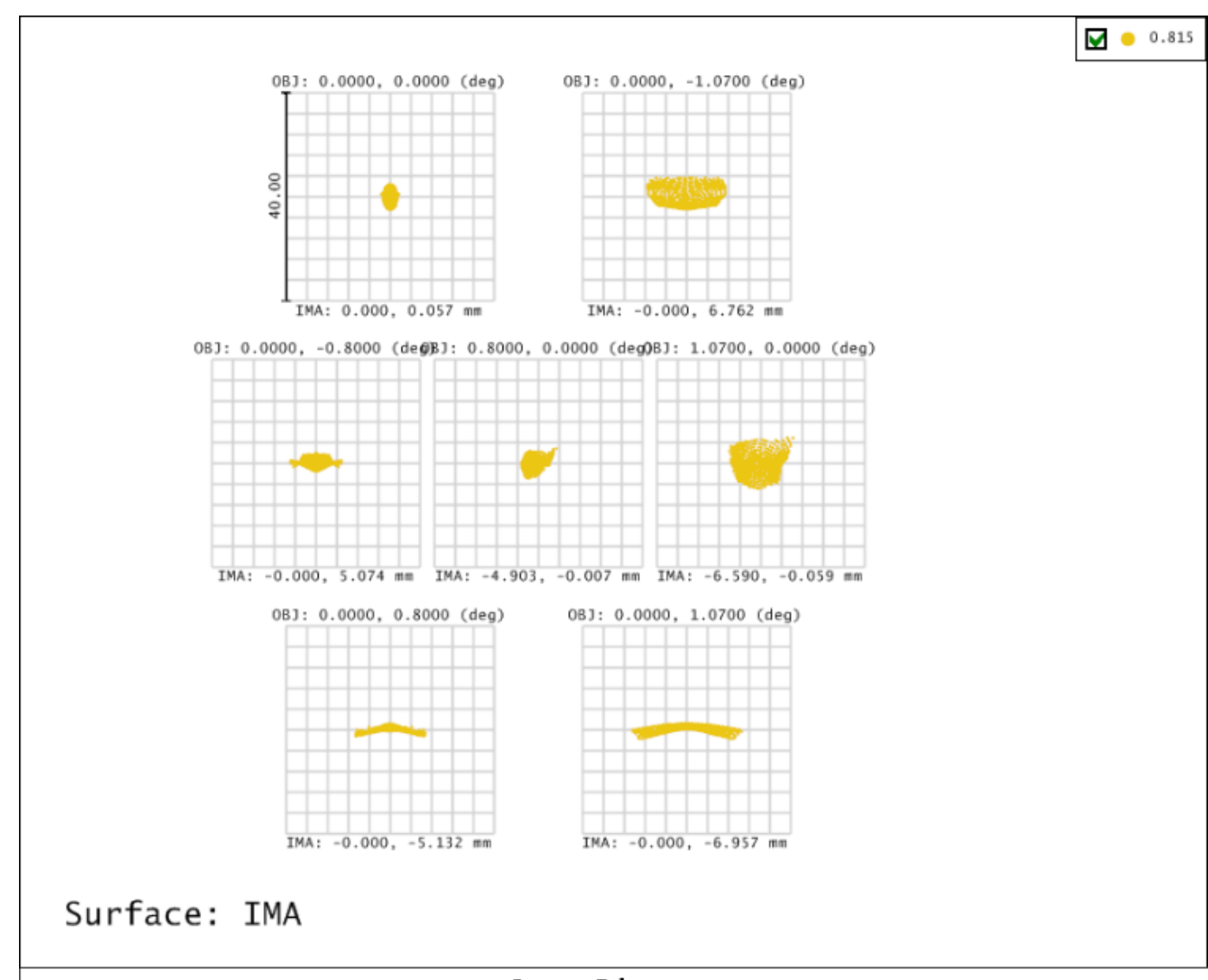

Spot Diagram

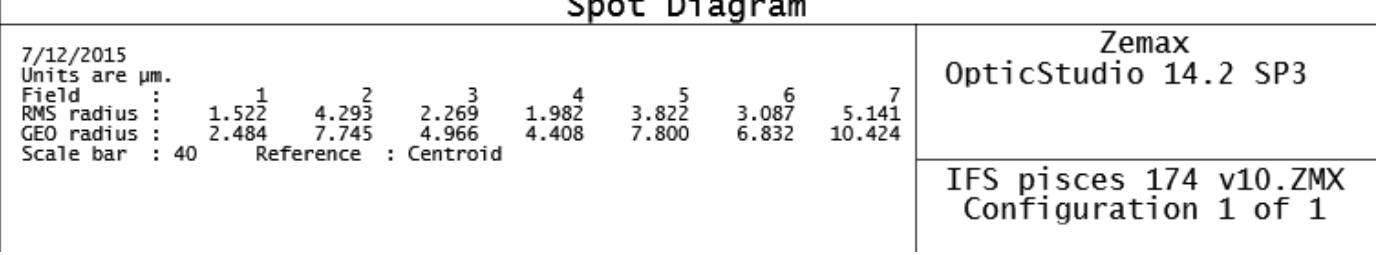

Spatial resolution: RMS spot size is less than detector pixel of $13 \mu \mathrm{m}$
Note: the result on this page is as-designed.

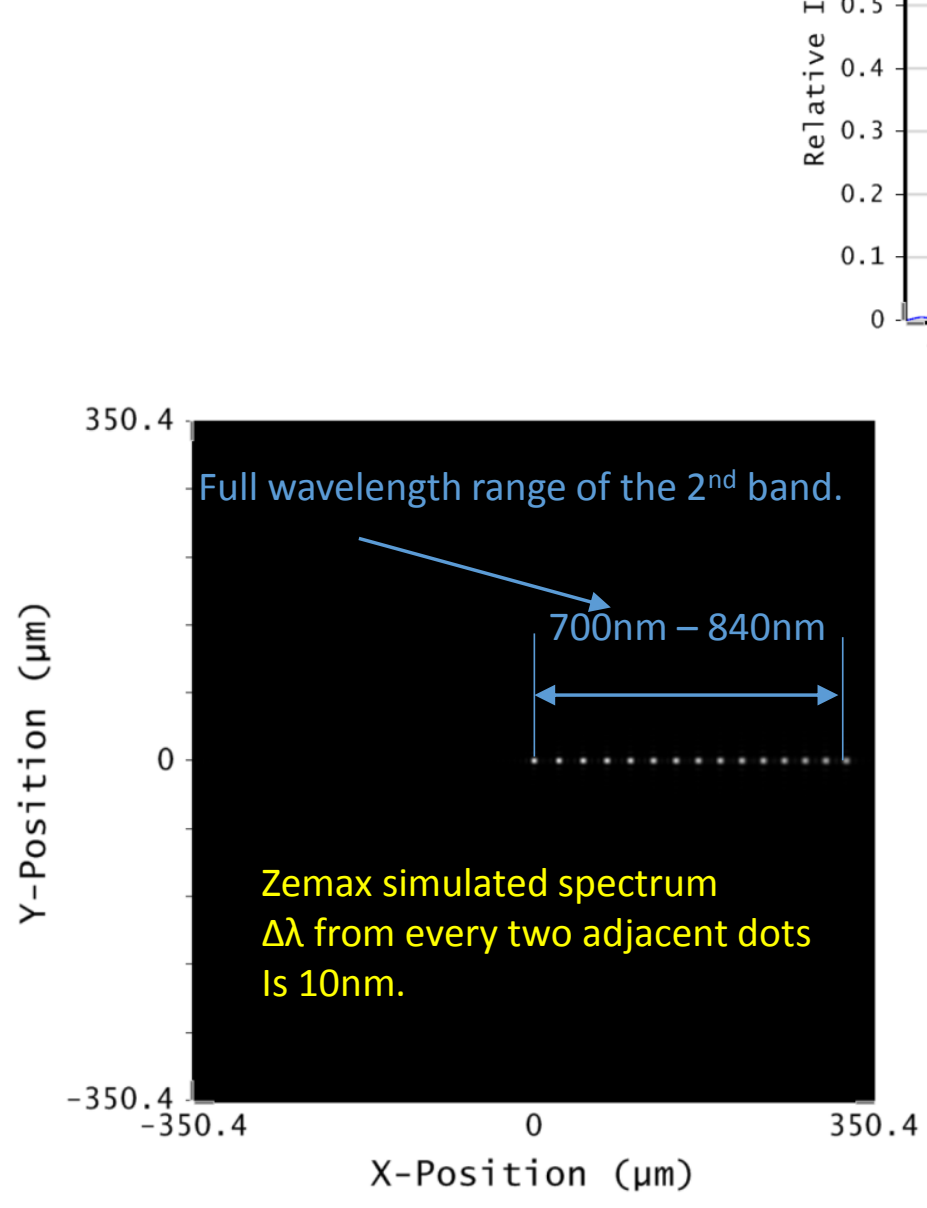

Spectral resolution meets

The required $\mathrm{R}=70$.
The 2 spectral lines at $\mathrm{R}=\lambda c / \Delta \lambda=70$ is well Separated. 


\section{Refractive}

\begin{tabular}{|c|c|c|c|}
\hline & & Pro & Con \\
\hline \multirow{4}{*}{$\begin{array}{l}\text { Perfor- } \\
\text { mance }\end{array}$} & Spot size & Refractive & Reflective \\
\hline & Distortion & Reflective & Refractive \\
\hline & $\begin{array}{l}X / Y \text { aspect } \\
\text { ratio }\end{array}$ & Refractive & Reflective \\
\hline & Throughput & Reflective & Refractive \\
\hline \multicolumn{2}{|l|}{ Volume } & Refractive & Reflective \\
\hline \multicolumn{2}{|c|}{ Fabrication } & Refractive & Reflective \\
\hline \multicolumn{2}{|c|}{ Alignment } & Refractive & Reflective \\
\hline \multicolumn{2}{|l|}{ Cost } & Refractive & Reflective \\
\hline
\end{tabular}

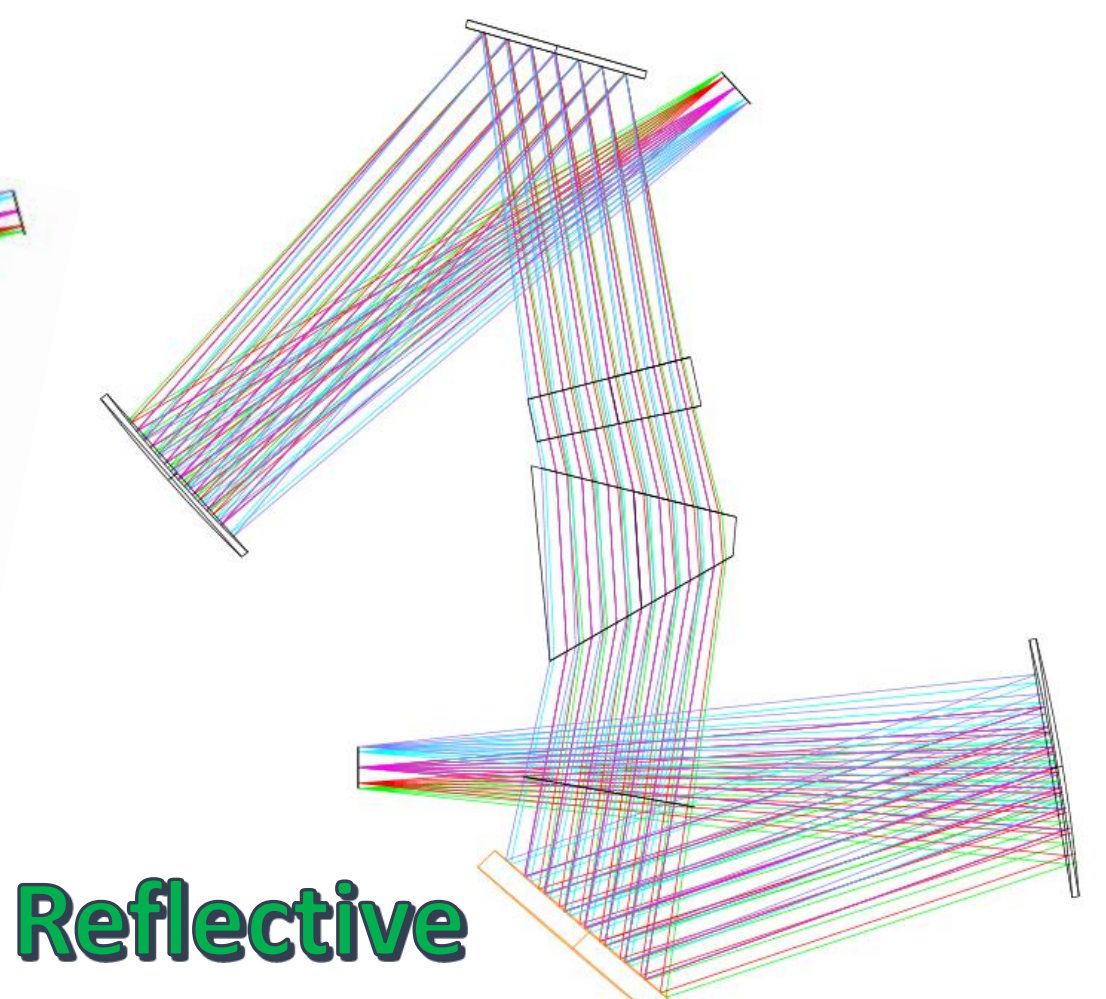

Two IFS designs use the same system specification: same f/\#, same wavelength range, same effective focal length, and same amount of spectral dispersion.

The envelop of reflective design is $\sim 350 \mathrm{~mm} \times 350 \mathrm{~mm}$.

The envelop for the refractive design is $\sim 610 \mathrm{~mm} \times 120 \mathrm{~mm}$.

The trade-off above is for PISCES wavelength range (600 nm to $970 \mathrm{~nm}$ ) only. For UV, mid and far IR, mirrors have to be used. 


\section{IFS Alignment Overview}

The basic alignment summary is the following:

1. To attach at least one alignment cube and one tooling ball nest to each element and sub-assembly.

2. To use theodolites, alignment telescopes, and Coordinate Measuring Machine (CMM) performing metrology of each element and sub-assembly to obtain the relation between the element and alignment references (cubes and nests).

3. To setup a Zemax alignment model to include the alignment references on all elements and subassemblies. To provide a table with all reference positions in a defined global coordinate.

4. To used metrology tools (theodolites and $\mathrm{CMM}$ ) aligning each element and sub-assembly to the pre-determined positions.

5. The interferometer will be used to measure the wavefront to verify the alignment at some key intermedium stages. 


\section{IFS Alignment Schematic}

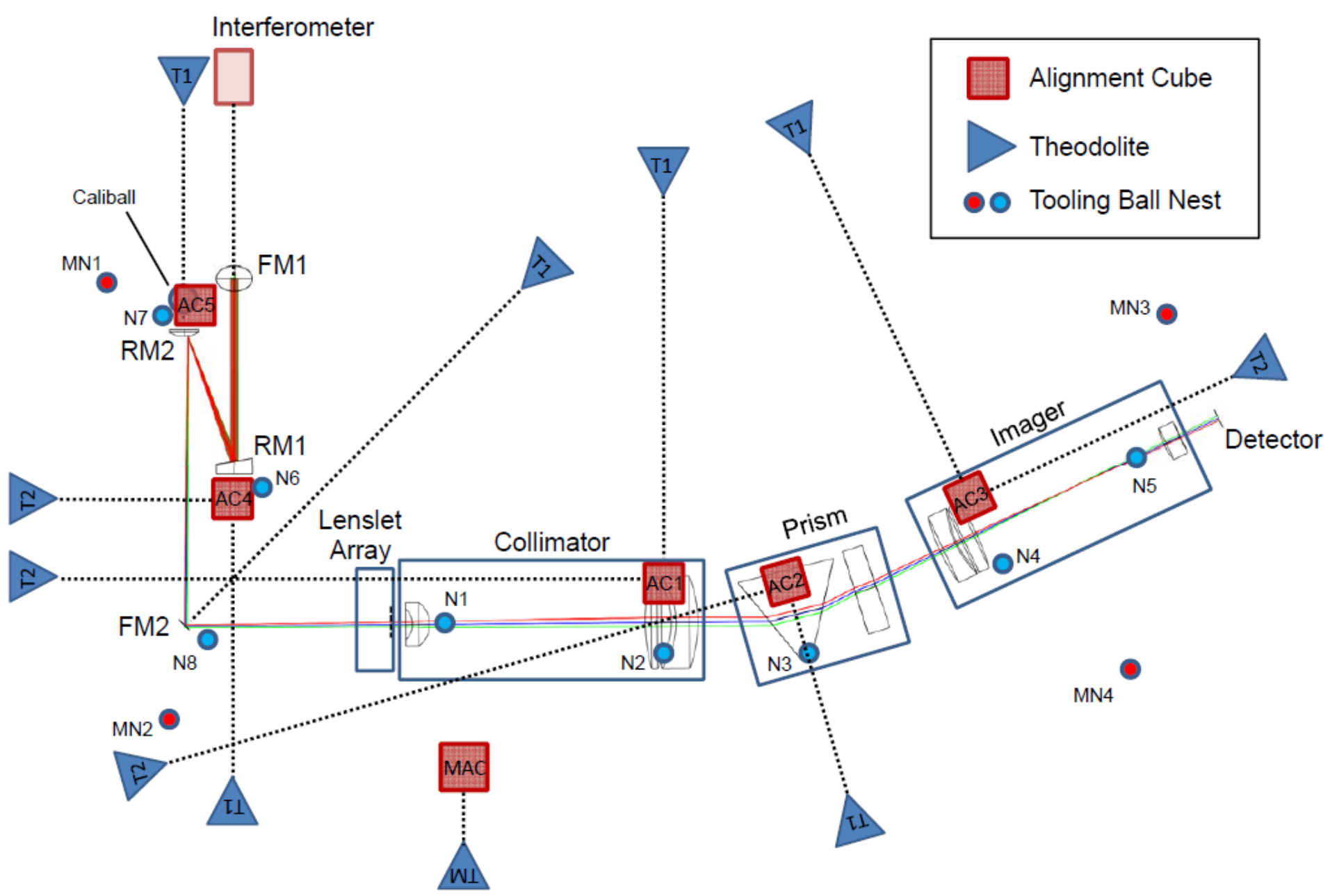




\section{Prototype Status}

- As mentioned in previous slides, the main advantage of the lenslet IFS is its simplicity and high throughput, the main disadvantage is the low efficiency of using detector pixels.

- Optical design was frozen.

- Relay optical elements have been procured and tested. The surface figures meet the specification. Ready for alignment.

- Lenslet array has been procured and tested.

- Spectrometer sub-assemblies, collimator, prism, and imager, have been contracted out to Photon Gear. Estimated delivery date is at the end of August.

- The mechanical design is completed, including the Photon Gear's sub-assemblies.

- Complete alignment plan is in place. Testbed simulator alignment is completed.

- Spectral and radiometric calibration plan is in progress. 


\section{Path Forward}

1. Evaluate the efficiency of using lenslet array to further compress the speckles.

2. Complete testbed simulator alignment and test.

3. Assemble and test PISCES.

4. Create a calibration plan: both spectral and radiometric.

5. Calibrate PISCES.

6. Deliver PISCES to JPL. 